\title{
Anesthesie van kittens een literatuuroverzicht met nadruk op de mogelijkheden in België
}

\author{
Anesthesia in kittens \\ a review of the literature with stress on the possibilities in Belgium
}

\author{
${ }^{1}$ E.H.K.A. Peeters, ${ }^{2}$ N. Porters, ${ }^{1}$ P.E.J. Bols, ${ }^{3}$ M. Nelissen, ${ }^{4}$ C.P.H. Moons, ${ }^{2}$ H. de Rooster, ${ }^{2}$ I. Polis \\ ${ }^{1}$ Laboratorium voor de Fysiologie en de Biochemie van de Huisdieren, Departement Diergeneeskundige \\ Wetenschappen, Universiteit Antwerpen, \\ Universiteitsplein 1, Gebouw U, B-2610 Wilrijk, België \\ ${ }^{2}$ Vakgroep Geneeskunde en Klinische Biologie van de Kleine Huisdieren, Faculteit Diergeneeskunde, \\ Universiteit Gent, Salisburylaan 133, B-9820 Merelbeke, België \\ ${ }^{3}$ Departement Biologie, Universiteit Antwerpen, \\ Groenenborgerlaan 171, Gebouw V, B-2020 Antwerpen, België \\ ${ }^{4}$ Vakgroep Voeding, Genetica en Ethologie, Faculteit Diergeneeskunde, Universiteit Gent, \\ Heidestraat 19, B-9820 Merelbeke \\ Els.Peeters2@ua.ac.be
}

\section{SAMENVATTING}

In België worden zelden electieve chirurgische ingrepen uitgevoerd bij zeer jonge kleine huisdieren. Veel dierenartsen hebben dan ook weinig ervaring met de anesthesie van pediatrische patiënten. In dit overzichtsartikel wordt kort ingegaan op de risicofactoren bij de anesthesie van kittens, gezien hun specifieke anatomie en fysiologie. Vervolgens wordt een overzicht gegeven van geschikte anesthetische protocollen. Injectieanesthesie op basis van combinaties met ketamine is een praktische, economische en efficiënte optie.

\begin{abstract}
In Belgium, elective surgical procedures in very young small animals are rarely performed. Consequently, most veterinarians have little experience in anesthetizing pediatric patients. This article describes potential problems of anesthesia in kittens, which are mainly linked to their specific anatomy and physiology. Additionally, some practical anesthetic protocols are highlighted. Combinations with ketamine are practical, economical and efficient options.
\end{abstract}

\section{INLEIDING}

Het anestheseren van pediatrische patiënten, zoals kittens, is in de meeste diergeneeskundige praktijken geen routine. Pediatrische patiënten worden immers meestal aangeboden voor dringende chirurgische ingrepen, zoals congenitale aandoeningen en trauma, en zelden voor electieve chirurgie, zoals vroegcastratie (Joyce en Yates, 2011). Door het gebrek aan ervaring is het verdoven van kittens voor veel dierenartsen een uitdaging. Daarom staan ze er vaak weigerachtig tegenover (Murray et al., 2008). Er is dus nood aan kennis van de mogelijkheden van anesthesie bij deze specifieke groep van patiënten. Hoewel in de wetenschappelijke literatuur verschillende protocollen beschreven zijn bij jonge kittens (6-16 weken), stroomt deze kennis slechts beperkt door naar de praktiserende dierenarts (Faggella en Aronsohn, 1993). In dit overzichtsartikel worden de belangrijkste aandachtspunten voor een veilige en efficiënte anesthesie en analgesie bij kittens besproken. Vervolgens wordt een overzicht gegeven van praktische anesthetische protocollen uit de literatuur.

\section{AANDACHTSPUNTEN BIJ DE JONGE PATIËNT}

De jonge patiënt verschilt op een aantal belangrijke punten met volwassen dieren. De meeste van deze verschillen zijn terug te voeren tot de anatomie van de kitten en de nog suboptimale functie van een aantal fysiologische mechanismen (Grundy, 2006; Holden, 2007) (Tabel 1). Uiteraard heeft dit niet enkel zijn weerslag op de technische uitvoering van een ingreep, maar ook op de farmacologische impact van de gebruikte anesthetica en analgetica (Meyer, 2007).

\section{Anatomie}

De anatomie van kittens brengt vooreerst technische moeilijkheden met zich mee. Voor het verdoven wordt bij voorkeur injectieanesthesie met behulp van intramusculaire en subcutane injecties toegepast maar ook gasanesthesie (Faggella en Aronsohn, 1993; Kustritz, 2002; Taylor, 2002). Gezien het kleine formaat en de beweeglijkheid van kittens is het plaatsen van een intraveneuze katheter niet evident. Ook het intuberen 
Tabel 1. Fysiologische normaalwaarden bij kittens (Naar Holden, 2007; Grundy, 2006; Nadeau en Colebatch, 1965; Moore, 1959).

\begin{tabular}{lcc}
\hline & Kitten (6 tot 16 weken) & Adult \\
\hline Rode bloedcellen $\left(\times 10^{6} / \mu \mathrm{L}\right)$ & $6,57-8,14$ & 8 \\
Witte bloedcellen $\left(\times 10^{3} / \mu \mathrm{L}\right)$ & $18,07-19,70$ & 21,9 \\
Hemoglobine $(\mathrm{g} / \mathrm{dL})$ & $9,1-11,0$ & 12,1 \\
Hematocriet $(\%)$ & $25-35$ & $29-45$ \\
Proteïne $(\mathrm{g} / \mathrm{dL})$ & $4,6-5,2$ & $5,8-8,0$ \\
Albumine $(\mathrm{g} / \mathrm{dL})$ & $2,2-2,4$ & $2,3-3,0$ \\
Cholesterol $(\mathrm{mg} / \mathrm{dL})$ & $222-434$ & $150-270$ \\
Glucose $(\mathrm{mg} / \mathrm{dL})$ & $99-112$ & $63-144$ \\
Bloeddruk $(\mathrm{mmHg})$ & $56-66$ & $100-150$ \\
Hartfrequentie $(\mathrm{slagen} / \mathrm{min})$ & $210-300$ & $140-220$ \\
Zuurstofconsumptie $\left(\mathrm{mL} / \mathrm{min}\right.$ per $\left.\mathrm{m}^{2}\right)$ & $200-870$ & 111,4 \\
Ademhalingsfrequentie $(\mathrm{ademhalingen} / \mathrm{min})$ & $20-36$ & $24-42$ \\
Rectale temperatuur $\left({ }^{\circ} \mathrm{C}\right)$ & 37,7 & $38,1-39,2$ \\
\end{tabular}

van kittens is niet altijd even eenvoudig en vergt meestal aangepast materiaal, zoals kleine tracheotubes met een interne diameter (ID) van 2-2,5 $\mathrm{mm}$, al dan niet voorzien van een cuff, alsook een smal laryngoscoopblad (bijvoorbeeld Miller nr. 0). Door het lage lichaamsgewicht (vaak slechts $1 \mathrm{~kg}$ ) is het zeer belangrijk om de dosering nauwkeurig af te stemmen. Het is aangeraden de kitten op ten minste $100 \mathrm{~g}$ nauwkeurig te wegen (Looney et al., 2008; Little, 2008). Het lage vetpercentage in het lichaam en het grotere percentage lichaamsvocht dan bij adulte dieren dragen ertoe bij dat anesthetica maar in beperkte mate opgeslagen kunnen worden en dus sneller herverdeeld worden (Aronsohn en Faggella, 1993; Stubbs et al., 1995; Taylor, 2002).

\section{Cardiovasculaire fysiologie}

De cardiovasculaire compensatiesystemen zijn bij kittens nog onderontwikkeld (Hutchinson et al., 1962; Sheridan et al., 1977; Chandler et al., 2008). Vooral de hartfrequentie is van belang om het volume rondgestuwd bloed constant te houden (hartdebiet = slagvolume $\mathrm{x}$ hartfrequentie), aangezien het hart van pediatrische patiënten slecht in staat is het slagvolume te verhogen (Lourenço en Ferreira, 2003; Chandler et al., 2008). Een daling van de hartfrequentie met een daarmee gepaard gaande afname van het hartdebiet, kan onvoldoende gecompenseerd worden door het opdrijven van de hartspiercontractiliteit (Davies et al., 1975; Meyer, 2007; Holden, 2007; Chandler et al., 2008). Bradycardie tijdens de anesthesie moet daarom zoveel mogelijk vermeden worden (Stubbs et al., 1995; Kustritz, 2002; Little, 2008). Ook het reguleren van de bloeddruk staat nog niet volledig op punt (zie verder), terwijl kittens van nature al een lagere bloeddruk hebben dan volwassen dieren (Hutchinson et al., 1962; Lourenço en Ferreira, 2003; Chandler et al., 2008) (Tabel 1). Het risico op hypotensie tijdens de narcose is daardoor groter bij kittens (Holden, 2007).

\section{Neurologie}

Het hierbovengenoemde verhoogde risico op bradycardie en hypotensie wordt mee veroorzaakt door de immaturiteit van het sympatisch zenuwstelsel (Grandy en Dunlop, 1991; Chandler et al., 2008). De effecten van het parasympathisch zenuwstelsel domineren en een verminderde controle van het vasomotorisch systeem is hiervan het gevolg (Holden, 2007; Chandler et al., 2008). Concreet houdt dit in dat de jonge dieren onvoldoende kunnen compenseren voor een plotse bloeddrukdaling, onder andere bij bloedverlies of tijdens anesthesie, omdat ze de perifere vaatweerstand onvoldoende kunnen opdrijven door vasoconstrictie.

\section{Respiratoire fysiologie}

Het zuurstofverbruik van kittens is ongeveer twee à drie maal groter dan dat van volwassen katten (Moore, 1959; Nadeau en Colebatch, 1965; Holden, 2007). Hun tidaal volume is echter ongeveer even groot (rond $8 \mathrm{~mL} / \mathrm{kg}$ ), zodat kittens een twee à drie maal hogere ademhalingsfrequentie moeten aanhouden om aan hun zuurstofbehoefte te kunnen voldoen $(\mathrm{Pa}-$ rot et al., 1984; Blanco et al., 1984; Mortola en Noworaj, 1985; Meyer, 2007; Little, 2008) (Tabel 1). Kittens kunnen zuurstoftekort niet compenseren door een inspiratoir reservevolume. Daarom is het zeer belangrijk dat de hoge ademhalingsfrequentie behouden kan blijven gedurende de anesthesie (Bonora et al., 1992; Stubbs et al., 1995; Kustritz, 2002; Taylor, 2002). Dit maakt ook dat kittens zeer kwetsbaar zijn voor zuurstoftekort, onder andere door obstructie van de luchtwegen door speeksel of braaksel (Haddad en Mellins, 1984; Hilaire en Duron, 1999; Meyer, 2007).

\section{Thermoregulatie}

Hypothermie is een van de belangrijkste risicofactoren bij het onder anesthesie brengen van kittens. Niet alleen is hun thermoregulatorisch systeem nog immatuur 
en hebben ze weinig isolerend onderhuids vet, ook hun grote oppervlakte/volumeratio maakt hen gevoelig voor onderkoeling (Kustritz, 2002; Little, 2008). Door de beperkte controle van het vasomotorisch systeem is ook de mogelijkheid tot perifere vasoconstrictie gelimiteerd, waardoor via de subcutane bloedvaten extra warmte verloren kan gaan (Holden, 2007). Hypothermie tijdens de narcose verhoogt het risico op bradycardie en hypotensie en vertraagt de eliminatie van anesthetica, met een trager herstel tot gevolg (Meyer, 2007).

\section{Hematologie}

Kittens hebben een lagere hemoglobineconcentratie en beginnen pas zelf bloed aan te maken vanaf de leeftijd van één maand (Meyers-Wallen et al., 1984) (Tabel 1). Samen met hun laag bloedvolume en lage bloeddruk betekent dit dat het verlies van enkele milliliters bloed al schadelijk kan zijn en eventueel zelfs anemie, hypotensie en hypoxie kan veroorzaken (Aronsohn en Faggella, 1993; Little, 2008). Bloedingen moeten dus zoveel mogelijk vermeden worden. Ook de plasmaproteïneconcentraties zijn lager dan bij adulten (Chandler et al., 2008) (Tabel 1). Van anesthetica met een sterke eiwitbinding blijft een grotere fractie ongebonden en effectief. Dit kan leiden tot een relatieve overdosage. Pas op een leeftijd van ongeveer acht weken worden volwassen plasmaalbumineniveaus gemeten (Meyer, 2007).

\section{Hepatisch systeem}

Afhankelijk van de leeftijd van de kitten is een aantal enzymatische systemen van de lever nog niet volledig operationeel. Dit heeft zijn invloed op de metabolisatie van farmaca, waardoor het effect van sommige anesthetica verlengd kan zijn. Pas rond de leeftijd van acht à twaalf weken wordt het hepatisch systeem als volledig functioneel beschouwd (Boothe en Tannert, 1992; Hosgood et al., 1998; Kustritz, 2002; Holden, 2007).

Naast de enzymatische afbraaksystemen is de lever mee verantwoordelijk voor het regelen van de bloedsuikerspiegel. Kittens zijn gevoeliger voor hypoglycemie dan volwassen katten omdat ze slechts kleine glycogeenreserves in de lever hebben en de glycogenolyse (omzetting van glycogeen tot glucose) en gluconeogenese (vorming van glucose uit niet-koolhydraten) traag verlopen (Kustritz, 2002; Holden, 2007; Little, 2008). Hypoglycemie kan daardoor niet snel gecompenseerd worden.

\section{Nieren}

De eerste zes tot acht weken na de geboorte zijn de nieren nog niet volledig ontwikkeld en is hun functie suboptimaal (Holden, 2007; Chandler et al., 2008). Ze worden gekenmerkt door een lage glomerulaire filtratiesnelheid, een beperkte plasmadoorstroming, een lage filtratiefractie en een beperkte mogelijkheid tot het concentreren van de urine (Pettifer en Grubb, 2007; Chandler et al., 2008). Hierdoor verloopt de excretie van anesthetica via de nieren trager dan bij volwassen dieren en worden de effecten ervan verlengd (Kustritz, 2002; Chandler et al., 2008; Little, 2008).

\section{ANESTHESIE}

Als er rekening gehouden wordt met de specifieke anatomische, fysiologische en farmacologische kenmerken en behoeften van de jonge patiënt, kan de anesthesie vlot en efficiënt verlopen. Het kan van levensbelang zijn voor de pediatrische patiënt om standaard een aantal maatregelen te implementeren in het protocol.

\section{Preoperatieve voorbereidingen}

Zoals altijd is een preanesthetisch klinisch onderzoek van de patiënt essentieel. Bij jonge dieren moet het gewicht nauwkeurig bepaald worden (tot 100 gram nauwkeurig) om tot een accurate dosering te komen (Looney et al., 2008). Kittens zijn gevoeliger voor de effecten van anesthetica dan volwassen katten en dienen dus een relatief lagere dosis toegediend te krijgen (Stubbs et al., 1995). Daarnaast is het lichaamsoppervlak bij kittens lichter dan $1,5 \mathrm{~kg}$ vaak een adequatere parameter dan het lichaamsgewicht om de dosering te bepalen (Tabel 2). Omwille van de grotere lichaamsoppervlakte tot lichaamsmassaratio, worden dergelijke kleine kittens namelijk makkelijk ondergedoseerd (Joyce en Yates, 2011). Om deze reden werd het Quad protocol ontwikkeld (Tabel 3), waarbij de dosering van de gebruikte farmaca gebaseerd wordt op de lichaamsoppervlakte (Joyce, 2010).

Door de kleine glycogeenreserves in de lever is het belangrijk de kittens niet te lang te laten vasten vóór een operatie. Als richtlijn wordt een kitten drie tot vier uur vóór een operatie voedsel onthouden (Holden, 2007; Pettifer en Grubb, 2007). Water blijft beschikbaar

Tabel 2. Berekening van dosering aan de hand van lichaamsoppervlakte.

\begin{tabular}{lcc}
\hline LG (kg) & LO $\left(\mathbf{m}^{2}\right)$ & $\begin{array}{c}\text { Toe te dienen volume } \\
\text { van elk product uit } \\
\text { het Quad Protocol }(\mathbf{m l})\end{array}$ \\
\hline 0,5 & 0,065 & 0,04 \\
0,6 & 0,074 & 0,04 \\
0,7 & 0,082 & 0,05 \\
0,8 & 0,090 & 0,05 \\
0,9 & 0,097 & 0,06 \\
1 & 0,104 & 0,06 \\
1,1 & 0,111 & 0,07 \\
1,2 & 0,118 & 0,07 \\
1,3 & 0,124 & 0,07 \\
1,4 & 0,130 & 0,08 \\
1,5 & 0,136 & 0,08 \\
\hline
\end{tabular}

Het gebruik van lichaamsoppervlakte (LO) i.p.v. lichaamsgewicht (LG) zorgt voor een adequatere dosering bij kittens onder 1,5 kg (Joyce en Yates, $2011)$. Voor de omzetting wordt de volgende formule gebruikt: $\mathrm{LO}=(10,4$ $\left.x \mathrm{LG}^{0,67}\right) / 100$ waarbij LO wordt uitgedrukt in $\mathrm{m}^{2}$ en LG in $\mathrm{kg}$. LO vermenigvuldigd met factor 0,6 geeft het volume dat nodig is van elk product uit het Quad Protocol. 
Tabel 3. Enkele praktische anesthetische protocollen uit de literatuur voor gebruik bij jonge dieren.

\begin{tabular}{|c|c|c|c|c|}
\hline Protocollen & Dosering & Toedieningswijze & Analgesie & Opmerkingen \\
\hline Atropine + & $0,04 \mathrm{mg} / \mathrm{kg}$ & IM & \multirow{4}{*}{ goed } & \multirow{7}{*}{$\begin{array}{l}\text { Bij voorkeur wordt } \\
\text { dexmedetomidine }(5-20 \mu \mathrm{g} / \mathrm{kg}) \\
\text { gebruikt i.p.v. medetomidine. }\end{array}$} \\
\hline Midazolam + & $0,22 \mathrm{mg} / \mathrm{kg}$ & IM & & \\
\hline Ketamine + & $5-10 \mathrm{mg} / \mathrm{kg}$ & IM & & \\
\hline Butorphanol & $0,44 \mathrm{mg} / \mathrm{kg}$ & IM & & \\
\hline Medetomidine + & $10-40 \mu \mathrm{g} / \mathrm{kg}$ & IM & & \\
\hline Ketamine + & $5 \mathrm{mg} / \mathrm{kg}$ & IM & goed & \\
\hline Butorphanol & $0,4 \mathrm{mg} / \mathrm{kg}$ & IV, IM of SC & & \\
\hline Medetomidine + & $600 \mu \mathrm{g} / \mathrm{m}^{2} *$ & IM & \multirow{4}{*}{$\begin{array}{l}\text { zeer goed tot } \\
6-12 \mathrm{u} \text { postoperatief }\end{array}$} & \multirow{7}{*}{$\begin{array}{l}\text { Dit is het Quad Protocol. } \\
\text { Gelijke volumes van elk } \\
\text { product worden hierbij toegediend. } \\
\text { I.p.v. medetomidine wordt echter } \\
\text { dexmedetomidine }(5-20 \mu \mathrm{g} / \mathrm{kg}) \text { aangeraden }\end{array}$} \\
\hline Ketamine + & $60 \mathrm{mg} / \mathrm{m}^{2}$ & IM & & \\
\hline Midazolam + & $3 \mathrm{mg} / \mathrm{m}^{2}$ & IM & & \\
\hline Buprenorphine & $180 \mu \mathrm{g} / \mathrm{m}^{2}$ & IM & & \\
\hline Tiletamine + Zolazepam & $11 \mathrm{mg} / \mathrm{kg}$ & IM & gemiddeld & \\
\hline Isofluraan & op effect & inhalatie & geen & \\
\hline Propofol & $4-12 \mathrm{mg} / \mathrm{kg}$ & IV & $\begin{array}{l}\text { afhankelijk van } \\
\text { premedicatie }\end{array}$ & \\
\hline
\end{tabular}

$\mathrm{IM}=$ intramusculair $\mathrm{IV}=$ intraveneus $\mathrm{SC}=$ subcutaan

*Voor de berekening van de dosering voor het Quad Protocol zie Tabel 2.

en indien de diertjes nog niet gespeend zijn, blijven ze tot vlak vóór de ingreep bij de kattin (Aronsohn en Faggella, 1993; Faggella en Aronsohn, 1993; Holden, 2007). De monitoring van het bloedglucosegehalte (normaalwaarden: 80-140 mg/dl) kan eenvoudig gebeuren aan de hand van een druppel bloed en een bloedglucosemeter (Chandler et al., 2008; Barton, 2009). Bij hypoglycemie (<60 mg/dl) kan er $50 \%$ glucose gedruppeld worden op de mondmucosa (Chandler et al., 2008; Koenig, 2009). Wanneer een intraveneuze lijn voorhanden is, kan er continu een glucose-infuus $(2,5-5 \%)$ toegediend worden tot normale bloedwaarden bereikt worden (Chandler et al., 2008).

Hypothermie kan voorkomen worden door slechts daar te scheren waar het nodig is, een alcoholvrije scrub op basis van een iodiumoplossing te gebruiken om afkoeling door verdamping te voorkomen, opgewarmde vloeistoffen te gebruiken, de kitten zo droog mogelijk te houden, de kitten te isoleren voor een maximaal behoud van warmte met onder andere overlevingsfolie en bubbeltjesplastic, gebruik te maken van een externe warmtebron, zoals infraroodlamp, Bair Hugger-warmtedeken, warmwatermatras, en door de lichaamstemperatuur te monitoren (Faggella en Aronsohn, 1993; Stubbs et al., 1995; Kustritz, 2002; Taylor, 2002; Little, 2008). Voorts is het aan te raden enkele producten bij de hand te houden die nuttig kunnen zijn bij het optreden van eventuele complicaties. Bloedverlies dient bijvoorbeeld onmiddellijk gecompenseerd te worden via de intraveneuze toediening van isotone vloeistoffen (Ringerlactaat of $\mathrm{NaCl} 0,9 \%$ ) al of niet met toegevoegde glucose (maximaal 5\%) (10ml/kg/uur) (Taylor, 2002; Chandler et al., 2008). Glucose 5\% in water mag niet gebruikt worden als enige infusie- vloeistof, omdat het aanleiding kan geven tot hypotone effecten en uitgesproken elektrolietafwijkingen (dilutie) (Koenig, 2009). Wanneer een intraveneuze toedieningsweg niet voorhanden is, kan er geopteerd worden voor het plaatsen van een intraosseuze naald. De plaatsingstechniek en het benodigde materiaal zijn uitvoerig beschreven (Giunti en Otto, 2009; Bukoski et al., 2010).

\section{Premedicatie}

Hoewel premedicatie niet in alle gevallen noodzakelijk is, is het gebruik ervan vaak toch voordelig omdat het een positieve bijdrage levert tot de anesthesiediepte, de spierrelaxatie en de analgesie tijdens de anesthesie. De meest gebruikte categorieën, ook bij jonge dieren, zijn anticholinergica, sedativa en analgetica. Anticholinergica, zoals atropine $(0,02-0,04$ $\mathrm{mg} / \mathrm{kg}$ ) en glycopyrrolaat $(0,01-0,02 \mathrm{mg} / \mathrm{kg})$ zijn beide antagonisten van het parasympathische zenuwstelsel (Backman et al., 1996; Dowling, 2005). Aangezien bij jonge kittens de effecten van het parasympathische zenuwstelsel domineren (Grandy en Dunlop, 1991; Chandler et al., 2008), kunnen deze anticholinergica als premedicatie gebruikt worden om cardiovasculaire depressie tegen te gaan (Buccino et al., 1966; Lehmann en Langer, 1982; Backman et al., 1996; Taylor, 2002; Dowling, 2005; Hennis en Leusink, 2007). Bovendien wordt ook de uitscheiding van secretieproducten in de luchtwegen hierdoor verminderd, wat obstructie van de luchtwegen helpt te voorkomen (Ueki et al., 1980; Grandy en Dunlop, 1991; Dowling, 2005; Hennis en Leusink, 2007). Meestal worden ze enkel toegediend wanneer er zich tijdens de anesthesie een acute brady- 
cardie voordoet. Ondanks zijn ietwat tragere aanzet, is er een lichte voorkeur voor het gebruik van glycopyrrolaat omdat het minder de neiging heeft om een sinustachycardie uit te lokken. Tranquillizers en sedativa, zoals benzodiazepinen (minor tranquillizers onder andere diazepam, midazolam, zolazepam), onderdrukken het centraal zenuwstelsel en hebben een synergistische werking met andere stoffen, zoals opioïden en ketamine, waarvan ze de effecten tevens verlengen (Taylor, 2002). Benzodiazepinen zijn relatief veilig in gebruik, aangezien ze slechts matige respiratoire en cardiovasculaire effecten, zoals vasodilatatie en hypotensie veroorzaken. Indien nodig kunnen ze worden tegengegaan met flumazenil (Taylor, 2002). Bij zeer jonge kittens geven ze een goede sedatie. Bij iets oudere dieren is een combinatie met andere sedativa en analgetica aangewezen om een goed effect te bekomen (Taylor, 2002). Ook fenothiazinen (major tranquillizers, onder andere acepromazine) bezitten sedatieve eigenschappen maar veroorzaken -net zoals de benzodiazepinen- geen analgesie (Johnstone, 2005). Het gebruik van fenothiazinen wordt afgeraden bij dieren jonger dan twaalf weken (Stubbs et al., 1995; Little, 2008). Ze veroorzaken cardiovasculaire effecten (door $\alpha$-lyse) die leiden tot vasodilatatie van het perifere vaatbed. Hypotensie en hypothermie kunnen het gevolg zijn (Grandy en Dunlop, 1991; Taylor, 2002; Khan, 2005; Pettifer en Grubb, 2007; Holden, 2007). Bovendien worden ze gemetaboliseerd in de lever. Daarbij kan de trage hepatische biotransformatie bij jonge dieren een verlengde onderdrukking van het centraal zenuwstelsel veroorzaken (Grandy en Dunlop, 1991).

De populaire $\alpha$-2-adrenerge agonisten, zoals (dex)medetomidine, bezitten uitgesproken sedatieve en spierrelaxerende eigenschappen en bieden daarnaast een goede viscerale analgesie (Grandy en Dunlop, 1991). Wanneer ze samen met andere stoffen gebruikt worden die het centraal zenuwstelsel onderdrukken, zorgen ze voor een gevoelige dosisvermindering van de daaropvolgende anesthetica. Omdat $\alpha$-2-adrenerge agonisten ernstige bradycardie en hypothermie kunnen veroorzaken, is voorzichtigheid geboden (Ponder en Clark, 1980; Myers et al., 1987; Stubbs et al., 1995; Cullen, 1996; Mogoa et al., 2001). Het toedienen van anticholinergica kan de bradycardie teniet doen, indien deze erg uitgesproken is. Bovendien is er een $\alpha$-2-antagonist (atipamezole) beschikbaar die de werking van de $\alpha$-2-adrenerge agonisten opheft, waardoor ze toch relatief veilig gebruikt kunnen worden bij kittens (Grandy en Dunlop, 1991; Taylor, 2002). Ze worden door de lever gemetaboliseerd en uitgescheiden via de nieren. Dit kan bij jonge dieren leiden tot een vertraagd herstel (Grandy en Dunlop, 1991; Taylor, 2002; Little, 2008). Daarom wordt bij voorkeur dexmedetomidine gebruikt, dat enkel de werkzame rechtsdraaiende en niet de overbodige linksdraaiende variant bevat, die voor een extra belasting van de lever en nieren zou zorgen (Macdonald et al., 1991; Kuusela et al., 2000; Granholm et al., 2006).

Narcotische analgetica, zoals butorfanol (met korte werkingsduur) en buprenorfine (met lange werkingsduur) zijn goede pijnstillers, die als premedicatie de vereiste dosis van de andere anesthetica verminderen (Grandy en Dunlop, 1991; Taylor, 2002; Little, 2008). Butorfanol geeft vooral een viscerale analgesie, terwijl buprenorfine zowel bij somatische als viscerale pijn kan gebruikt worden (Stanway et al., 2002; Robertson, 2008). De effecten van butorfanol op het cardiovasculair systeem en het ademhalingsstelsel zijn normaal beperkt. Het gebruik in combinatie met andere stoffen die het centraal zenuwstelsel onderdrukken, versterkt echter de effecten van butorfanol, waardoor een onderdrukking van het cardiorespiratoire systeem kan plaatsvinden (Dyson, 1990; Sawyer, 1998; Hall et al., 1999). Het toedienen van anticholinergica is dan aangeraden. Hoewel butorfanol voornamelijk in de lever gemetaboliseerd wordt, wordt zelden een verlengde onderdrukking van het centraal zenuwstelsel waargenomen (Grandy en Dunlop, 1991; Taylor, 2002; Little, 2008). Zuivere $\mu$-agonisten, zoals onder andere morfine en methadone, kunnen gebruikt worden bij meer uitgesproken pijn maar hebben doorgaans meer dosisgerelateerde cardiorespiratoire nevenwerkingen (bradycardie, hypoventilatie, ...), hetgeen bij kittens het beste vermeden wordt (Mathews, 2005; Steagall et al., 2006; Mathews, 2008). Deze effecten kunnen tegengegaan worden door het gebruik van naloxone (Mathews, 2005). Ook niet-steroïdale ontstekingsremmers (NSAID's) kunnen gebruikt worden als pijnstiller, maar enkel bij kittens ouder dan acht weken met een mature nierfunctie en met een dosisvermindering van 50 tot $75 \%$ (Holden, 2007). NSAID's moeten sowieso voorzichtig gebruikt worden bij katten omwille van hun lage hepatische glucuronidatiecapaciteit. Daarom gaat de voorkeur naar meloxicam, dat gemetaboliseerd wordt door oxidatieve enzymen (Lascelles et al., 2007).

\section{Inductie en onderhoud}

Voor de inductie en het onderhoud van de anesthesie zijn verscheidene opties voorhanden, die ook verschillen in wijze van toediening. Het gemakkelijkst toe te dienen zijn de dissociatieve anesthetica, zoals ketamine, die intramusculair (IM) gegeven kunnen worden. Ze worden vaak gebruikt in combinatie met benzodiazepinen om een betere spierrelaxatie te verkrijgen (Verstegen et al., 1989; Grandy en Dunlop, 1991; Taylor, 2002) en met parasympathicolytica om de bronchiale en speekselsecretie en aldus het risico op aspiratie te verminderen (Hosgood, 2001; Little, 2008). Dissociatieve anesthetica veroorzaken over het algemeen slechts een geringe respiratoire depressie en zorgen voor een indirecte stimulatie van het cardiovasculair systeem (Taylor, 2002). Ondanks hun relatieve veiligheid is er nog geen bijkomend onderzoek gepubliceerd over het gebruik bij dieren jonger dan acht weken. Het gebruik bij deze jonge dieren dient dus met omzichtigheid te gebeuren. De beperkte hepatische metabolisatie bij de kat zorgt ervoor dat ketamine onveranderd wordt uitgescheiden via de nieren, wat bij dergelijke jonge dieren een vertraagd herstel tot gevolg kan hebben (Grandy en Dunlop, 1991; Hosgood et al., 1998; Little, 2008). 
Propofol heeft op het vlak van metabolisatie minder nadelen dan ketamine. Hoewel de metabolisatie behalve in de longen ook voor een stuk in de lever en de nieren plaatsvindt, worden bij menselijke patiënten met nier- en leveraandoeningen geen veranderingen in het farmacokinetisch profiel vastgesteld (Biebuyk, 1989; Grandy en Dunlop, 1991). Propofol is reeds met goed gevolg gebruikt als anestheticum bij pups en iets oudere katten (acht maanden) (Grandy en Dunlop, 1991; Stubbs et al., 1995) en kan vrij veilig gebruikt worden bij kittens vanaf acht weken mits de emulsie langzaam wordt ingespoten over een periode van één à twee minuten (Hosgood et al., 1998; Hosgood, 2001). Propofol kan zowel voor inductie als onderhoud worden gebruikt en induceert een snelle, kortdurende anesthesie met goede spierrelaxatie maar met geringe analgesie (Hall et al., 1999; Hosgood, 2001; Johnstone, 2005). Het wordt daarom steeds in combinatie met analgetica gebruikt (Grandy en Dunlop, 1991; Hosgood et al., 1998). Propofol is vooral geschikt voor korte procedures, aangezien een langdurige toediening ( $>30$ minuten) een vertraagd herstel met algemene malaise tot gevolg kan hebben (Hall et al., 2001; Pascoe et al., 2006; Nadaf et al., 2007; Bester, 2009; Riviere en Papich, 2009). Tot de nadelige effecten van propofol behoren een dosisafhankelijke depressie van het cardiorespiratoire systeem (Saint-Maurice, 1991; Hall et al., 1999; Nadaf et al., 2007; Riviere en Papich, 2009; Drobatz en Costello, 2010). Het grootste praktische nadeel van propofol bij kittens is de intraveneuze (IV) toediening (Grandy en Dunlop, 1991; Stubbs et al., 1995; Hosgood, 2001).

Voor de jongste patiënten zijn inhalatieanesthetica, zoals isofluraan en sevofluraan, de beste keuze (Holden, 2007). Isofluraan is het enige geregistreerde inhalatieanestheticum in België voor diergeneeskundig gebruik. Het is het inhalatieanestheticum bij voorkeur bij dieren jonger dan tien weken omdat het bijna volledig ongemetaboliseerd $( \pm 0,2 \%$ metabolisatie tegenover $2-5 \%$ voor sevofluraan) weer wordt uitgeademd en aldus het immature hepatorenale systeem niet belast (Martis et al., 1981; Grandy en Dunlop, 1991; Stubbs et al., 1995; Little, 2008). Zowel isofluraan als sevofluraan geeft een dosisgebonden cardiovasculaire en respiratoire depressie en biedt de mogelijkheid om de anesthesiediepte nauwkeurig en permanent aan te passen (Hikasa et al., 1997; Pypendop en Ilkiw, 2004).

Een goede premedicatie op basis van een narcotisch analgeticum, zoals butorfanol, kan nuttig zijn om de benodigde dosis te verlagen met behoud van voldoende pijnstilling (Grandy en Dunlop, 1991; Kustritz, 2002; Taylor, 2002; Grint et al., 2009). Het nadeel van inhalatieanesthetica is de omslachtigere manier van toediening. Voor de inductie, die bij jonge patiënten veel sneller verloopt dan bij adulte katten, wordt meestal gebruik gemaakt van een goed passend masker (Kustritz, 2002; Meyer, 2007; Little, 2008). Hierbij dient rekening gehouden te worden met mogelijke contaminatie van de omgeving. Voor het onderhoud van de anesthesie is een aangepast systeem met endotracheale intubatie aangewezen (bijvoorbeeld Bain-circuit, T-stukje van Ayre, pediatrisch cirkelsysteem). Bij het intuberen kunnen kleine endotracheale tubes $(2,0-3,5 \mathrm{~mm}$ interne diameter) worden gebruikt, maar deze moeten regelmatig (elke 30 minuten) worden gecontroleerd op permeabiliteit (Grandy en Dunlop, 1991; Stubbs et al., 1995). Bij dieren met een lichaamsgewicht van minder dan $5 \mathrm{~kg}$ wordt bij voorkeur een non-rebreathing-circuit gebruikt (Grandy en Dunlop, 1991; Stubbs et al., 1995; Taylor, 2002). Non-rebreathing-systemen maken geen gebruik van $\mathrm{CO}_{2}$-absorbers, maar van hoge verse gasflows om de uitgeademde $\mathrm{CO}_{2}$ uit het systeem te verwijderen. De voordelen van deze systemen zijn hun gebruiksgemak, een beperkte ademweerstand, een minimale mechanische dode ruimte en de mogelijkheid tot snelle aanpassing van de anesthesiediepte (Hartsfield, 2007). Indien er tijdens de anesthesie hypoventilatie optreedt, kan de beademing van het patiëntje voorzichtig manueel of met behulp van een ventilator ondersteund worden.

Het monitoren van jonge patiënten is van essentieel belang. Hierbij dient extra aandacht besteed te worden aan een respiratoire depressie en/of obstructie van de luchtwegen, aangezien het zeer belangrijk is dat de hoge ademhalingsfrequentie van kittens behouden kan blijven gedurende de anesthesie (Holden, 2007). Ook het behoud van de lichaamstemperatuur en een hoge hartfrequentie is erg belangrijk. Het gebruik van hulpmiddelen, zoals polsoximeter, capnograaf en ECG, is aan te bevelen. Een oesofagale stethoscoop is eveneens bruikbaar om de adem- en hartgeluiden te evalueren en is niet duur (Aronsohn en Faggella, 1993) (Figuur 1).

\section{Enkele voorbeeldprotocollen uit de literatuur}

De doseringen voor de hieronder besproken protocollen en enkele andere praktische protocollen worden weergegeven in Tabel 3.

Een veelgebruikt protocol is een combinatie van atropine, midazolam, ketamine en butorfanol. In deze combinatie zorgt midazolam voor een minimale sedatie en een verbeterde spierrelaxatie om de verhoogde spiertonus als gevolg van ketamine tegen te gaan (Grandy en Dunlop, 1991). Atropine ondersteunt de hartfrequentie en vermindert de respiratoire en speekselsecretie om het risico op aspiratie te beperken (Grandy en Dunlop, 1991). Butorfanol biedt bijkomende analgesie (Faggella en Aronsohn, 1993; Little, 2008). Als onderhoudsanestheticum kan eventueel bijkomend isofluraan gebruikt worden. Deze combinatie is geschikt voor intensievere operaties (Grandy en Dunlop, 1991; Faggella en Aronsohn, 1993; Howe, 1999; Taylor, 2002). Het voordeel van deze combinatie is het beperkte effect op het cardiovasculair en respiratoir systeem (Stubbs et al., 1995; Taylor, 2002).

Een andere combinatie met ketamine is deze met medetomidine en butorfanol. Metedomidine gebruikt als premedicatie in een lage dosis maakt de combinatie geschikt voor jongere kittens (Verstegen et al., 1989; Grandy en Dunlop, 1991; Taylor, 2002). Wanneer een minder oppervlakkig anesthesieniveau gewenst is, wordt isofluraan gebruikt als onderhoudsanestheticum.

Recentelijk werd ook het Quad Protocol ontwik- 


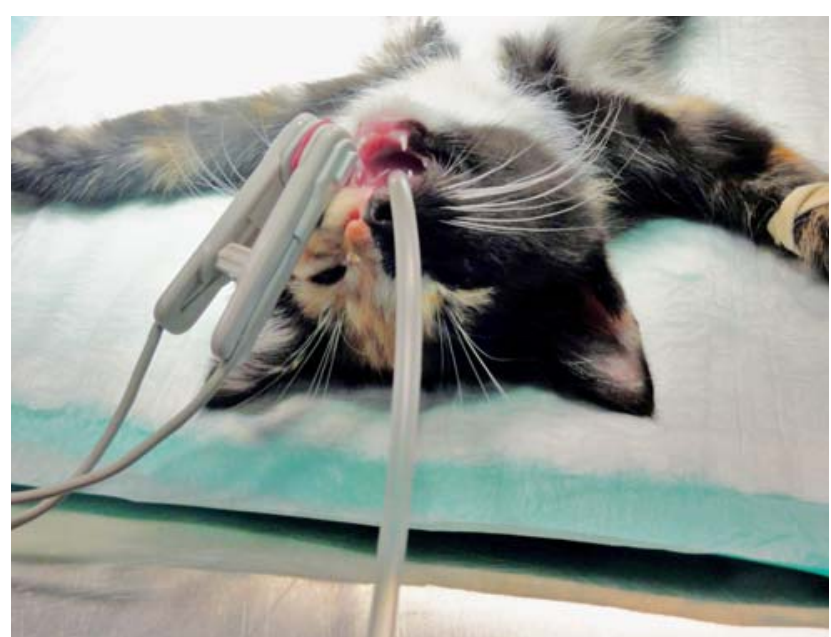

Figuur 1. Monitoring van een geanestheseerde kitten met behulp van een oesofagale stethoscoop en polsoximeter.

keld, dat het probleem van onderdosering bij kittens met een kleine lichaamsmassa (voornamelijk lichter dan 1,5 $\mathrm{kg}$ ) ondervangt (Joyce, 2010). De dosering wordt gebaseerd op de lichaamsoppervlakte in plaats van op het lichaamsgewicht (Tabel 2). De gebruikte farmaca in dit protocol zijn medetomidine, ketamine, midazolam en buprenorfine. Een ander voordeel van dit protocol is dat alle farmaca in gelijke volumes worden toegediend in een enkelvoudige injectie (Joyce en Yates, 2011) (Tabel 2 en 3$)$.

De combinatie tiletamine-zolazepam is in België verkrijgbaar in poedervorm met oplosmiddel. Het geeft een goede inductie en myorelaxatie, hoewel -net als bij ketamine- spontane bewegingen en verhoogde spiertonus kunnen voorkomen (Grandy en Dunlop, 1991; Faggella en Aronsohn, 1993). Het nadeel van dit preparaat is de beperkte houdbaarheid, eens het opgelost is. Deze combinatie kan al gebruikt worden vanaf een leeftijd van zes weken (Faggella en Aronsohn, 1993; Howe, 1999). Afhankelijk van de dosis duurt de anesthesie tussen vijftien en veertig minuten (Grandy en Dunlop, 1991). Indien nodig kan isofluraan toegediend worden (Stubbs et al., 1995; Howe, 1999), wat echter wel een trager herstel kan opleveren (Faggella en Aronsohn, 1993).

\section{Postoperatieve zorgen}

Tijdens het herstel na de anesthesie vergen jonge patiënten extra zorg en aandacht. Het controleren van de respiratie blijft een belangrijk punt, net als het voorkomen en behandelen van hypothermie. Pediatrische patiënten hebben nood aan een extra warmtebron tijdens de recovery (Aronsohn en Faggella, 1993). Dit kan een warmwaterkruik, een elektrisch dekentje, een Bair Hugger-warmtedeken of infraroodlamp zijn, maar helemaal ideaal is een incubator met toegevoegde zuurstof (Haskins, 1981). Om een te forse daling in de bloedsuikerspiegel tegen te gaan, kunnen glucose houdende vloeistoffen per os worden toegediend (Aronsohn en Faggella, 1993; Taylor, 2002). Verder is het aan te raden om de kittens binnen het uur na het ontwaken uit de anesthesie een kleine portie hoog energetische voeding te geven of bij de moeder te brengen zodat ze kunnen drinken (Aronsohn en Faggella, 1993; Faggella en Aronsohn, 1993; Kustritz, 2002; Little, 2008).

\section{CONCLUSIE}

Hoewel jonge dieren bijzonder kwetsbaar zijn, is het -mits het in acht nemen van enkele risicofactorengoed mogelijk een kitten op een veilige manier onder narcose te brengen. De kitten moet warm gehouden worden, het mag niet te lang vasten, de respiratie moet goed gevolgd worden en men moet alert zijn voor bradycardie. Er zijn verschillende anesthetische en analgetische protocollen beschreven die beantwoorden aan de noden in verschillende omstandigheden. Voor de dagdagelijkse praktijk zijn combinaties met ketamine een praktische, economische en efficiënte optie.

\section{LITERATUUR}

Aronsohn M. G., Faggella A. M. (1993). Surgical techniques for neutering 6- to 14-week-old kittens. Journal of the American Veterinary Medical Association 202, 5355.

Backman S., Stein R., Blank D., Collier B., Polosa C. (1996). Different properties of the bradycardia produced by neostigmine and edrophonium in the cat. Canadian Journal of Anesthesia / Journal Canadien d'Anesthésie 43, 731-740.

Barton L. (2009). Daily assessment of the critically ill patient. In: D.Silverstein, K. Hopper (Editors). Small Animal Critical Care Medicine. Saunders Elsevier, St. Louis, p. 852-855.

Bester L. (2009). Pharmacokinetics of Propofol in Cats. MMedVet dissertation, University of Pretoria.

Biebuyk J. F. (1989). Propofol: a new intravenous anesthetic. Anesthesiology 71, 260-277.

Blanco C. E., Hanson M. A., Johnson P., Rigatto H. (1984). Breathing pattern of kittens during hypoxia. Journal of Applied Physiology 56, 12-17.

Bonora M., Boule M., Gautier H. (1992). Diaphragmatic and ventilatory responses to alveolar hypoxia and hypercapnia in conscious kittens. Journal of Applied Physiology 72, 203-210.

Boothe D. M., Tannert K. (1992). Special considerations for drug and fluid therapy in the pediatric patient. Compendium on Continuing Education for the Practicing Veterinarian 14, 313-329.

Buccino R. A., Sonnenblick E. H., Cooper T. H. E. O., Braunwald E. U. G. E. (1966). Direct positive inotropic effect of acetylcholine on myocardium. Circulation Research 19, 1097-1108.

Bukoski A., Winter M., Bandt C., Wilson M., Shih A. (2010). Original study: comparison of three intraosseous access techniques in cats. Journal of Veterinary Emergency and Critical Care 20, 393-397.

Chandler E. A., Gaskell R. M., Gaskell C. J. (2008). Feline Medicine and Therapeutics. John Wiley \& Sons.

Cullen L. K. (1996). Medetomidine sedation in dogs and cats: a review of its pharmacology, antagonism and dose. British Veterinary Journal 152, 519-535.

Davies P., Dewar J., Tynan M., Ward R. (1975). Post-natal developmental changes in the length-tension relationship 
of cat papillary muscles. The Journal of Physiology 253, 95-102.

Dowling P. M. (2005). Pharmacology. In: C.M.Kahn, S. Line (Editors). The Merck Veterinary Manual. 9 ed., Merck \& Co. Inc., Whitehouse Station, N.J., USA, p. 2036.

Drobatz K. J., Costello M. (2010). Feline Emergency and Critical Care Medicine. John Wiley \& Sons.

Dyson D. H. (1990). Update on butorphanol tartrate: use in small animals. Canadian Veterinary Journal 31, 120-121.

Faggella A. M., Aronsohn M. G. (1993). Anesthetic techniques for neutering 6- to 14-week-old kittens. Journal of the American Veterinary Medical Association 202, 5662.

Giunti M., Otto C. M. (2009). Intraosseous catheterization. In: D.Silverstein, K. Hopper (Editors). Small Animal Critical Care Medicine. Saunders Elsevier, St. Louis, p. 263-266.

Grandy G. L., Dunlop C. I. (1991). Anesthesia of pups and kittens. Journal of the American Veterinary Medical Association 198, 1244-1249.

Granholm M., McKusick B. C., Westerholm F. C., Aspegren J. C. (2006). Evaluation of the clinical efficacy and safety of dexmedetomidine or medetomidine in cats and their reversal with atipamezole. Veterinary Anaesthesia and Analgesia 33, 214-223.

Grint N. J., Burford J., Dugdale A. H. (2009). Investigating medetomidine-buprenorphine as preanaesthetic medication in cats. Journal of Small Animal Practice 50, 73-81.

Grundy S. A. (2006). Clinically relevant physiology of the neonate. Veterinary Clinics of North America-Small Animal Practice 36, 443.

Haddad G. G., Mellins R. B. (1984). Hypoxia and respiratory control in early life. Annual Review of Physiology 46, 629-643.

Hall L. W., Clarke K. W., Trim C. M. (2001). Veterinary Anaesthesia. W.B. Saunders.

Hall T. L., Duke T., Townsend H. G., Caulkett N. A., Cantwell S. L. (1999). The effect of opioid and acepromazine premedication on the anesthetic induction dose of propofol in cats. Canadian Veterinary Journal 40, 867870.

Hartsfield S. M. (2007). Anesthetic Machines and breathing systems. In: W.J.Tranquilli, J. C. Thurmon, K. A. Grimm (Editors). Lumb and Jones' Veterinary Anesthesia and Analgesia. 4 ed., Blackwell Pub., Ames, Iowa, USA, p. 453-494.

Haskins S. C. (1981). Hypothermia and its prevention during general anesthesia in cats. American Journal of Veterinary Research 42, 856-861.

Hennis P. J., Leusink J. A. (2007). Anesthesiology. 2 ed., Bohn Stafleu van Loghum, Houten, The Netherlands.

Hikasa Y., Ohe N., Takase K., Ogasawara S. (1997). Cardiopulmonary effects of sevoflurane in cats: comparison with isoflurane, halothane, and enflurane. Research in Veterinary Science 63, 205-210.

Hilaire G., Duron B. (1999). Maturation of the mammalian respiratory system. Physiological Reviews 79, 325-360.

Holden D. (2007). Paediatric Patients. In: C.Seymour, T. Duke-Novakovski (Editors). Manual of Canine and Feline Anaesthesia and Analgesia. BSAVA, Gloucester, UK, p. 296-302.

Hosgood G. (2001). Anesthesia and Surgery. In: J.D.Hoskins (Editor). Veterinary pediatrics: dogs and cats form birth to six months. 3 ed., Elsevier Health Sciences, Burlington, USA, p. 525-547.

Hosgood G., Hoskins J. D., Davidson J., Smith J. A. (1998).
Small Animal Paediatric Medicine and Surgery. Butterworth-Heinemann Ltd., Oxford, UK.

Howe L. M. (1999). Prepuberal gonadectomy in dogs and cats - part I. Compendium on Continuing Education for the Practicing Veterinarian 21, 103-111.

Hutchinson E. A., Percival C. J., Young I. M. (1962). Development of cardiovascular responses in the kitten. Experimental Physiology 47, 201-210.

Johnstone I. (2005). Pharmacology. In: C.M.Kahn, S. Line (Editors). The Merck Veterinary Manual. 9 ed., Merck \& Co. Inc., Whitehouse Station, N.J., USA, p. 2026.

Joyce, A. (2010). A novel anaesthetic protocol for the neutering of kittens 6-16 weeks of age. In: Proceedings of the 53rd Annual Congress BSAVA. 08-11 april 2010, Birmingham, UK.

Joyce A., Yates D. (2011). Help stop teenage pregnancy!: Early-age neutering in cats. Journal of Feline Medicine and Surgery 13, 3-10.

Khan S. A. (2005). Toxicology. In: C.M.Kahn \& S. Line (Editors). The Merck Veterinary Manual. 9 ed., Merck \& Co. Inc., Whitehouse Station, N.J., USA, p. 2535.

Koenig A. (2009). Hypoglycemia. In: D.Silverstein, K. Hopper (Editors), Small Animal Critical Care Medicine. Saunders Elsevier, St. Louis, p. 295-298.

Kustritz M. V. (2002). Early spay-neuter: clinical considerations. Clinical Techniques in Small Animal Practice 17, 124-128.

Kuusela E., Raekallio M., Anttila M., Falck I., Mölsä S., Vainio O. (2000). Clinical effects and pharmacokinetics of medetomidine and its enantiomers in dogs. Journal of Veterinary Pharmacology and Therapeutics 23, 15-20.

Lascelles B. D., Court M., Hardie E. M., Robertson S. A. (2007). Nonsteroidal anti-inflammatory drugs in cats: a review. Veterinary Anaesthesia and Analgesia 34, 228-250.

Lehmann J., Langer S. Z. (1982). Muscarinic receptors on dopamine terminals in the cat caudate nucleus: Neuromodulation of $[3 \mathrm{H}]$ dopamine release in vitro by endogenous acetylcholine. Brain Research 248, 61-69.

Little S. (2008). Early Age Spay and Neuter in the Cat. Dr.Susan Little's Website, Online Source. http://catvet.homestead.com/EarlyAlter.html

Looney A. L., Bohling M. W., Bushby P. A., Howe L. M., Griffin B., Levy J. K. et al. (2008). The Association of Shelter Veterinarians veterinary medical care guidelines for spay-neuter programs. Journal of the American Veterinary Medical Association 233, 74-86.

Lourenço M. L. G., Ferreira H. (2003). Electrocardiographic evolution in cats from birth to 30 days of age. Canadian Veterinary Journal 44, 914-917.

Macdonald E., Scheinin M., Scheinin H., Virtanen R. (1991). Comparison of the behavioral and neurochemical effects of the 2 optical enantiomers of medetomidine, a selective alpha-2-adrenoceptor agonist. Journal of Pharmacology and Experimental Therapeutics 259, 848-854.

Martis L., Lynch S., Napoli M. D., Woods E. F. (1981). Biotransformation of sevoflurane in dogs and rats. Anesthesia and Analgesia 60, 186-191.

Mathews K. A. (2008). Pain management for the pregnant, lactating, and neonatal to pediatric cat and dog. Veterinary Clinics of North America-Small Animal Practice 38, 1291+ .

Mathews K. A. (2005). Analgesia for the pregnant, lactating and neonatal to pediatric cat and dog. Journal of Veterinary Emergency and Critical Care 15, 273-284.

Meyer R. E. (2007). Anesthesia of pediatric small animal patients. In: R.D.Gleed \& J. W. Ludders (Editors). Recent 
Advances in Veterinary Anesthesia and Analgesia: Companion Animals. IVIS, Ithaca, NY.

Meyers-Wallen V. N., Haskins M. E., Patterson D. F. (1984). Hematologic values in healthy neonatal, weanling and juvenile kittens. American Journal of Veterinary Research 45, 1322-1327.

Mogoa E. G., Mbithi P. M., Nguhiu J. M., Kihurani D. (2001). Alpha-2-adrenoceptor agonists in veterinary practice. Kenya Veterinarian 22, 82-90.

Moore R. E. (1959). Oxygen consumption and body temperature in new-born kittens subjected to hypoxia and reoxygenation. Journal of Physiology-London 149, 500518.

Mortola J., Noworaj a. (1985). Breathing pattern and growth: comparative aspects. Journal of Comparative Physiology B: Biochemical, Systemic, and Environmental Physiology 155, 171-176.

Murray J. K., Skillings E., Gruffydd-Jones T. J. (2008). Opinions of veterinarians about the age at which kittens should be neutered. Veterinary Record 163, 381-385.

Myers R. D., Beleslin D. B., Rezvani A. H. (1987). Hypothermia: role of alpha-1- and alpha-2-noradrenergic receptors in the hypothalamus of the cat. Pharmacology Biochemistry and Behavior 26, 373-379.

Nadaf H., Avizeh R., Raazi Jalali M., Rasekh H., Mohammad Khani Z. (2007). Effects of propofol on the arterial blood pressure, blood gases and vital signs of cat. Scientific-Research Iranian Veterinary Journal 3, 95-102.

Nadeau R. A., Colebatch H. J. H. (1965). Normal respiratory and circulatory values in the cat. Journal of Applied Physiology 20, 836-838.

Parot S., Bonora M., Gautier H., Marlot D. (1984). Developmental changes in ventilation and breathing pattern in unanesthetized kittens. Respiration Physiology 58, 253262.

Pascoe P. J., Ilkiw J. E., Frischmeyer K. J. (2006). The effect of the duration of propofol administration on recovery from anesthesia in cats. Veterinary Anaesthesia and Analgesia 33, 2-7.

Pettifer G. R., Grubb T. L. (2007). Neonatal and geriatric patients. In: W.J.Tranquilli, J. C. Thurmon, K. A. Grimm (Editors), Lumb \& Jones' Veterinary Anesthesia and Analgesia. 4 ed., Blackwell Publishing, Ames, Iowa, p. 985991.
Ponder S. W., Clark W. G. (1980). Prolonged depression of thermoregulation after xylazine administration to cats. Journal of Veterinary Pharmacology and Therapeutics 3, 203-207.

Pypendop B. H., Ilkiw J. E. (2004). Hemodynamic effects of sevoflurane in cats. American Journal of Veterinary Research 65, 20-25.

Riviere J. E., Papich M. G. (2009). Veterinary Pharmacology and Therapeutics. Wiley-Blackwell.

Robertson S. A. (2008). Managing pain in feline patients. Veterinary Clinics of North America-Small Animal Practice 38, 1267.

Saint-Maurice C. (1991). Propofol in pediatric anesthesia. Cahiers d'Anesthésiology 39, 411-420.

Sawyer D. C. (1998). Pain control in small-animal patients. Applied Animal Behaviour Science 59, 135-146.

Sheridan D. J., Cullen M. J., Tynan M. J. (1977). Postnatal ultrastructural changes in the cat myocardium: a morphometric study. Cardiovascular Research 11, 536-540.

Stanway G. W., Taylor P. M., Brodbelt D. C. (2002). A preliminary investigation comparing pre-operative morphine and buprenorphine for postoperative analgesia and sedation in cats. Veterinary Anaesthesia and Analgesia 29, 29-35.

Steagall P. V. M., Carnicelli P., Taylor P. M., Luna S. P. L., Dixon M., Ferreira T. H. (2006). Effects of subcutaneous methadone, morphine, buprenorphine or saline on thermal and pressure thresholds in cats. Journal of Veterinary Pharmacology and Therapeutics 29, 531-537.

Stubbs W. P., Salmeri K. R., Bloomberg M. S. (1995). Early neutering of the dog and cat. In: J.D.Bonagura (Editor). Kirk's Current Veterinary Therapy XII. 12 ed., W.B. Saunders Company, Philadelphia, p. 1037-1040.

Taylor P. (2002). Anaesthesia for Neutering Kittens. FAB Journal 40, 22-24.

Ueki I., German V. F., Nadel J. A. (1980). Micropipette measurement of airway submucosal gland secretion. Autonomic effects. The American review of respiratory disease 121, 351-357.

Verstegen J., Fargetton X., Ectors F. (1989). Medetomidine/ketamine anaesthesia in cats. Acta Veterinaria Scandinavica Supplementum 85, 117-123. 